\title{
Aminoquinoline Fluorescent Labels Obstruct Efficient Removal of $N$-Glycan Core $\alpha(1-6)$ Fucose by Bovine Kidney $\alpha$-L-Fucosidase (BKF)
}

\author{
Róisín O’Flaherty, ${ }^{*} \dagger \odot$ Aoife M. Harbison, ${ }^{\ddagger}$ Philip J. Hanley, ${ }^{\ddagger}$ Christopher H. Taron, ${ }^{\S}$ Elisa Fadda, ${ }^{\ddagger}$
} and Pauline M. Rudd ${ }^{\dagger}$

${ }^{\dagger}$ NIBRT GlycoScience Group, National Institute for Bioprocessing, Research and Training, Foster's Avenue, Mount Merrion,
Blackrock, Co., Dublin, Ireland
${ }^{\ddagger}$ Department of Chemistry, Maynooth University, Maynooth, Kildare Ireland
${ }^{\S}$ New England Biolabs, Ipswich, Massachusetts 01938, United States

Supporting Information

ABSTRACT: Here we report evidence that new aminoquinoline $N$-glycan fluorescent labels interfere with the release of core $\alpha(1-$ 6) fucose from $N$-glycans by bovine kidney $\alpha$-L-fucosidase (BKF). $\mathrm{BKF}$ is a commonly employed exoglycosidase for core $\alpha(1-6)$ fucose determination. Molecular simulations of the bound and unbound Fuc- $\alpha(1-6)$-GlcNAc, where GlcNAc is situated at the reducing end for all $\mathrm{N}$-glycans, suggest that the reduced BKF activity may be due to a nonoptimal fit of the highest populated conformers in the BKF active binding site at room temperature. Population analysis and free energy estimates suggest that an enhanced flexibility of the labeled sugar, which facilitates recognition and binding, can be achievable with extended reaction conditions. We provide these experimental conditions
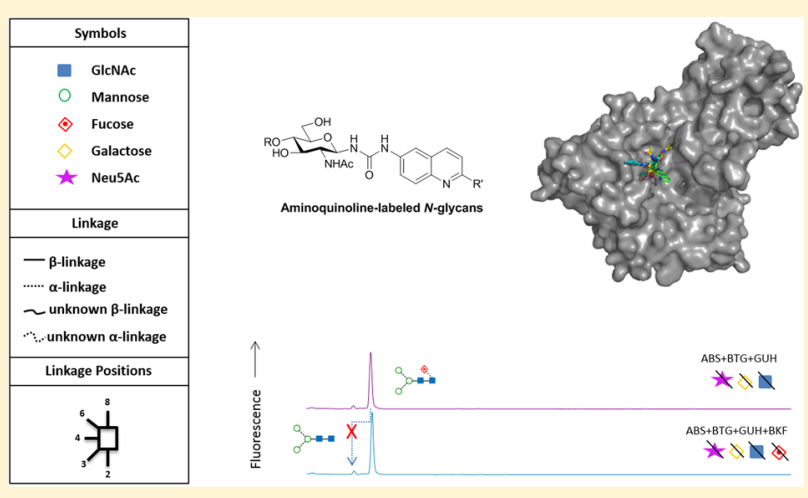
using a sequential exoglycosidase digestion array using high concentrations of BKF.

KEYWORDS: aminoquinoline (AQC), bovine kidney fucosidase (BKF), N-glycan, ultra performance liquid chromatography (UPLC)

\section{INTRODUCTION}

$N$-Glycan fucosylation, with fucose linked via $\alpha(1-2), \alpha(1-3)$, $\alpha(1-4), \alpha(1-6)$ linkages to $\mathrm{Gal}$ or GlcNAc residues, plays major roles in plants, mammals, and other biological systems and therefore can be exploited as tools for biomarker discovery. ${ }^{1,2}$ They play important roles in cellular recognition processes including blood transfusion reactions, selectinmediated leukocyte-endothelial adhesion, and host-microbe interactions. ${ }^{2}$ Fucosylation is a critical quality attribute for characterization of biotherapeutics such as monoclonal antibodies and is becoming increasingly important for product solubility, stability, pharmacokinetics, and immunogenicity studies as well as for the regulation of safe and efficacious drugs. $^{3}$

$N$-Glycan analysis of glycoproteins involves glycoprotein purification, denaturation, and enzymatic release of $\mathrm{N}$-glycans using Peptide- $\mathrm{N}$-Glycosidase F (PNGase F). ${ }^{4}$ The $\mathrm{N}$-glycans can be fluorescently and stoichiometrically labeled with 2aminobenzamide (2-AB) and quantified using (ultra)-high performance liquid chromatography (HPLC/UPLC), ${ }^{4}$ electrophoresis or by coupled liquid chromatography mass spectrometry techniques. ${ }^{5}$ Exoglycosidase digestion assays ${ }^{6}$ in combination with databases such as Glycobase ${ }^{7}$ are performed to structurally elucidate the glycans. In these arrays, individual glycosidases remove terminal sugars with specificity for a particular type of sugar, its anomeric configuration $(\alpha$ or $\beta)$, and its linkage to an adjacent sugar. For elucidation of $N$-glycan core $\alpha(1,6)$ fucose, the enzyme bovine kidney fucosidase (BKF) $\alpha(1-2,3,4,6)$ is most commonly used and has been successfully employed on various different substrates including $\mathrm{N}$-glycans on glycoproteins $\mathrm{IgG}^{6}{ }^{6} \mathrm{IgM},{ }^{8}$ and O-glycans, ${ }^{9}$ respectively.

New labels have recently been developed for improved fluorescent labeling of released $N$-glycans for structural analyses. Recently we described the use of an aminoquinoline carbamate fluorescent label, 6-aminoquinolyl-N-hydroxysuccinimidyl carbamate (AQC) in place of the traditional 2$\mathrm{AB}$ label (see Figure 1). ${ }^{10}$ This glycan label gives a 20 -fold improvement in fluorescent detection, compared to 2-AB. Additionally, Waters' RapiFluor-MS, also an aminoquinoline glycan label, significantly overcomes the low MS ionization efficiency of $2-\mathrm{AB}$ and increases sensitivity beyond 100-fold (see Figure 1). ${ }^{11}$ Despite the obvious analytical advances aminoquinoline labels represent, they have also dramatically altered the ease at which $\mathrm{N}$-glycan core fucose can be

Received: August 15, 2017

Published: September 27, 2017 


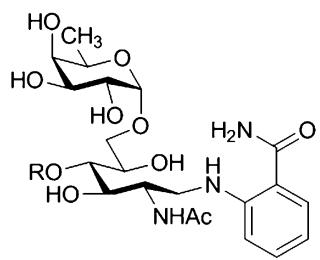

2-AB-labeled $\mathbf{N}$-glycans with core a(1-6) fucose

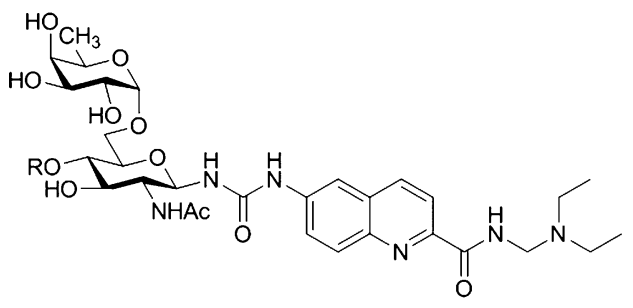

Rapifluor-MS-labeled $\mathbf{N}$-glycans with core a(1-6) fucose

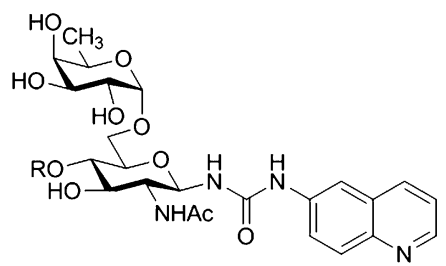

AQC-labeled $\mathbf{N}$-glycans with core a(1-6) fucose

Figure 1. 2-AB-, RapiFluor-MS-, and AQC-labeled N-glycans structures with core $\alpha(1-6)$ fucose. The $\mathrm{R}$ group represents any fluorescently labeled $\mathrm{N}$-glycans and indicates the extension occurs via $\beta(1-4)$ glycosidic linkage to the $N$-acetyl glucosamine.

enzymatically verified. In the present study, we show that BKF has a significantly reduced ability to remove core $\alpha(1,6)$ fucose from both AQC- or RapiFluor-MS-labeled N-glycans. We further present molecular simulation of BKF's active site to explore the possible basis for its inability to efficiently act in the presence of this new family of labels. We provide optimized exoglycosidase digestion array conditions using stringent conditions for the successful characterization of $\mathrm{N}$-glycans.

\section{MATERIALS AND METHODS}

\subsection{Materials and Chemistry}

All chemical reagents and solvents were purchased from SigmaAldrich (St. Louis, MO). Human IgG was purchased from Sigma-Aldrich (I4506) for reduction, alkylation, and $\mathrm{N}$-glycan labeling with $2-\mathrm{AB}$ and $\mathrm{AQC}$ as sources for 2-AB- and AQClabeled IgG N-glycans. Waters RapiFluor-MS glycan performance standard (186007983) was used as a source of RapiFluorMS-labeled IgG N-glycans. Recombinant PNGase F (P0709L), ABS $(\alpha 2-3,6,8,9$ Neuraminidase A, P0722L, $20 \mathrm{mU} / \mathrm{mL})$, BTG (bovine testes $\beta(1-3 / 4)$-galactosidase, $2 \mathrm{mU} / \mathrm{mL}$ ), GUH $(\beta$-N-Acetylglucosaminidase S, $4 \mathrm{mU} / \mathrm{ml})$, and BKF $(\alpha 1-$ 2,3,4,6 Fucosidase, $2 \mathrm{mU} / \mathrm{mL}$ ) were obtained from New England Biolabs (Ipswich, Massachusetts). BTG (PZGKX5013, $5 \mathrm{U} / \mathrm{mL}$ ), BKF (PZGKX-5006, $500 \mathrm{mU}$ ), and JBM (PZGKX-5010, $150 \mathrm{U} / \mathrm{mL}$ ) were purchased from Prozyme (San Leandro, California). 10K Nanosep centrifugal devices were purchased from Pall (Port Washington, NY) and Hypersep Diol cartridges from Thermo Fischer Scientific
(Waltham, Massachusetts). Samples were analyzed on a Waters Acquity H-Class UPLC instrument (Milford, MA).

\subsection{Glycan Nomenclature}

Colored Oxford nomenclature is used throughout, which provides embedded linkage information. ${ }^{12}$ Structure abbreviations: all $\mathrm{N}$-glycans have two core GlcNAcs; $\mathrm{F}$ at the start of the abbreviation indicates a core-fucose $\alpha(1,6)$ linked to the inner GlcNAc; $M x$, number ( $x$ ) of mannose on core GlcNAcs; Ax, number of antenna (GlcNAc) on trimannosyl core; A2, biantennary with both GlcNAcs as $\beta(1,2)$ linked; B, bisecting GlcNAc linked $\beta(1,4)$ to $\beta(1,4)$ mannose; $\mathrm{Gx}$, number (x) of $\beta(1,4)$ linked galactose on antenna; $\mathrm{Sx}$, number $(\mathrm{x})$ of sialic acids linked to galactose.

\subsection{Preparation of 2-AB-Labeled $\mathrm{N}$-Glycans from Human IgG}

A pool of released $\mathrm{N}$-glycans from human IgG was prepared for subsequent fluorescent labeling for visualization by UPLC chromatography. For eight separate vials, reduction of human IgG (525 $\mu \mathrm{g}$ in $100 \mu \mathrm{L}$ phosphate buffer per vial, $\mathrm{pH} 7.4)$ was afforded by addition of dithiothreithol (DTT, $40 \mu \mathrm{L}$ per vial, $100 \mathrm{mM}$ ) by incubation at $700 \mathrm{rpm}$ at $65{ }^{\circ} \mathrm{C}$ for $45 \mathrm{~min}$. Alkylation was afforded by treatment with iodoacetamide (IAA, $40 \mu \mathrm{L}$ per vial, $20 \mathrm{mM}$ ) by incubation at rt for $1 \mathrm{~h}$. PNGase $\mathrm{F}$ (25 $\mu \mathrm{L}$ per vial) was added and incubated at $37{ }^{\circ} \mathrm{C}$ overnight. The eight $10 \mathrm{~K}$ Nanosep centrifugal devices were preequilibrated with 50:50 water:acetonitrile, spun at $7000 \mathrm{rpm}$ for $10 \mathrm{~min}$, and the supernatants were discarded. The $\mathrm{N}$-glycan mixtures were added to each spin cartridge and centrifuged at $12000 \mathrm{rpm}$ for $2 \mathrm{~min}$ collecting the purified supernatants. The filters were washed with $800 \mu \mathrm{L}$ of water by centrifugation at $12000 \mathrm{rpm}$ for $10 \mathrm{~min}$, and the supernatants were pooled. Each vial was pooled and vacuum-dried to give a stock solution of unlabeled IgG $\mathrm{N}$-glycans. A portion of the unlabeled glycans were dissolved in 2-AB labeling mixture $(350 \mathrm{mM} 2-\mathrm{AB}, 1 \mathrm{M}$ $\left.\mathrm{NaCNBH}_{3} \mathrm{AcOH}: \mathrm{DMSO} 30: 70\right)$, incubated at $70{ }^{\circ} \mathrm{C}$ at 700 rpm for $2 \mathrm{~h}$. The reaction was quenched with $200 \mu \mathrm{L}$ of $95 \%$ MeCN. SPE Clean-up was performed using pre-equilibrated HyperSep Diol cartridges (washing with $1 \mathrm{~mL} 95 \% \mathrm{MeCN}$, $100 \% \mathrm{H}_{2} \mathrm{O}$ and $95 \% \mathrm{MeCN}$ respectively), whereby the supernatant was transferred to the HyperSep Diol cartridges, washed with $2 \times 1 \mathrm{~mL}$ of $95 \% \mathrm{MeCN}$, followed by elution with $2 \times 1 \mathrm{~mL}$ of $20 \% \mathrm{MeCN}$. The supernatant was collected and dried for UPLC chromatography.

\subsection{Preparation of AQC-Labeled N-Glycans from Human $\lg G$}

AQC-labeled $\mathrm{N}$-glycans from IgG were denatured, alkylated, and AQC-labeled according to the literature reference. ${ }^{13}$

2.5. Standard Exoglycosidase Digestion Conditions for 2-AB-, RapiFluor-MS-, and AQC-Labeled IgG N-Glycans

All enzymes were obtained from NEB ((Ipswich, Massachusetts). The 2-AB-, RapiFluor-MS-, and AQC-labeled glycans were digested in a volume of $10 \mu \mathrm{L}$ for $18 \mathrm{~h}$ at $37^{\circ} \mathrm{C}$ in $50 \mathrm{mM}$ sodium acetate buffer, $\mathrm{pH} 5.5$ using arrays of the following enzymes: ABS $(\alpha 2-3,6,8,9$ Neuraminidase A, P0722L, final concentration $2 \mathrm{mU} / \mathrm{mL}$ ), BTG (bovine testes $\beta(1-3 / 4)$ galactosidase, final concentration $400 \mathrm{U} / \mathrm{mL})$, GUH $(\beta-N$ Acetylglucosaminidase $S$, final concentration $800 \mathrm{U} / \mathrm{ml}$ ), and BKF $(\alpha 1-2,3,4,6$ Fucosidase, final concentration $800 \mathrm{U} / \mathrm{mL})$. After incubation, enzymes were removed by filtration through $10 \mathrm{kDa}$ protein-binding EZ filters (Millipore Corporation). $\mathrm{N}$ Glycans were then analyzed by HILIC UPLC Chromatography. 
Supporting Information (SI) Table S-1 contains the exoglycoside digestion array panel.

\subsection{Optimized Exoglycosidase Digestion Conditions Using} AQC-Labeled IgG1 N-Glycans

All enzymes were obtained from either NEB ((Ipswich, Massachusetts) or Prozyme ((San Leandro, California). The AQC-labeled glycans were digested in a volume of $10 \mu \mathrm{L}$ in a sequential addition for 4 days at $37{ }^{\circ} \mathrm{C}$ in $50 \mathrm{mM}$ sodium acetate buffer, $\mathrm{pH} 5.5$ using arrays of the following enzymes: ABS $(\alpha 2-3,6,8,9$ Neuraminidase A, PZGK80040, final concentration $0.5 \mathrm{U} / \mathrm{mL}$ ), BTG (bovine testes $\beta(1-3 / 4)$ galactosidase, PZGKX-5013, final concentration $1 \mathrm{U} / \mathrm{mL}$ ), GUH $(\beta$-N-Acetylglucosaminidase S, P0744L, final concentration $800 \mathrm{U} / \mathrm{mL})$, BKF $(\alpha 1-2,3,4,6$ Fucosidase, PZGKX5006, final concentration $4 \mathrm{U} / \mathrm{mL}), \mathrm{JBM}((\alpha 1-2,3,6$ Mannosidase J, PZGKX-5010, final concentration $60 \mathrm{U} / \mathrm{mL}$ ). After incubation, enzymes were removed by filtration through $10 \mathrm{kDa}$ protein-binding EZ filters (Millipore Corporation). $\mathrm{N}$ Glycans were then analyzed by HILIC UPLC Chromatography. SI Table S-2 contains the exoglycosidase digestion array panel.

\subsection{Ultra Performance Liquid Chromatography (UPLC)} with Fluorescence Detection (FLD)

Separation of 2-AB- and aminoquinoline-labeled $N$-glycans was carried out by UPLC with fluorescence detection on a Waters ACQUITY UPLC H-Class instrument consisting of a binary solvent manager, sample manager and fluorescence detector under the control of Empower 3 software (Waters, Milford, MA). The HILIC separations were performed using a Waters Ethylene Bridged Hybrid $(\mathrm{BEH})$ Glycan column $(150 \times 2.1$ $\mathrm{mm}$ i.d. 186004742, $1.7 \mu \mathrm{m} \mathrm{BEH}$ particles) with $50 \mathrm{mM}$ ammonium formate $(\mathrm{pH} 4.4)$ as solvent $\mathrm{A}$ and $\mathrm{MeCN}$ as solvent B. The column was fitted with an ACQUITY in-line 0.2 $\mu \mathrm{m}$ filter. The separation was performed using a linear gradient of $70-53 \% \mathrm{MeCN}$ at $0.56 \mathrm{~mL} / \mathrm{min}$ in 30 and $16.5 \mathrm{~min}$, respectively, for 2-AB- and aminoquinoline-labeled $N$-glycans. An injection volume of $10 \mu \mathrm{L}$ prepared in $70 \% \mathrm{v} / \mathrm{v} \mathrm{MeCN}$ was used throughout. Samples were maintained at $5{ }^{\circ} \mathrm{C}$ prior to injection, and the separation temperature was $40{ }^{\circ} \mathrm{C}$. The FLD excitation/emission wavelengths were $\lambda \mathrm{ex}=330 \mathrm{~nm}(2-\mathrm{AB})$, $245 \mathrm{~nm}$ (aminoquinline) and $\lambda \mathrm{em}=420 \mathrm{~nm}(2-\mathrm{AB}), 395 \mathrm{~nm}$ (aminoquinoline), respectively. The system was calibrated using an external standard of hydrolyzed and 2-AB-labeled glucose oligomers to create a dextran ladder, as described previously. ${ }^{14}$ A fifth-order polynomial distribution curve was fitted to the dextran ladder to assign glucose unit (GU) values from retention times (using Empower software from Waters).

\subsection{Computational Methods}

Because of the different chemical nature of the groups in the fluorescently labeled Fuc- $\alpha(1-6)$-GlcNAc, we used a combination of empirical force fields to run the $\mathrm{MD}$ calculations, namely, GLYCAM06-h1 ${ }^{15}$ for the sugar moiety, and the Generalized AMBER Force Field (GAFF) ${ }^{16}$ for the fluorescent labels and for the open GlcNAc in the 2-AB tagged glycan. The structures of the open GlcNAc, AQC, and 2-AB labels were generated and saved in PDB format using the academic version of Schrödinger Maestro v.10.7.015. ${ }^{17}$ The point charges for these groups have been calculated with USCF Chimera v.1.11.2, ${ }^{18}$ and were manually rescaled to guarantee the neutrality of the whole system. A library file of GAFF parameters and a frcmod file were generated using the tleap module of AMBER 12. ${ }^{19}$ Topology file and coordinate files for the labeled glycans were also generated with tleap and solvated with the TIP $3 \mathrm{P}^{20}$ water model. To ensure exhaustive conformational sampling, four different starting conformations of the $\alpha(1-6)$ glycosidic linkage, which included $g t, g g$, and $t g$ conformers, were used as starting points for the MD simulations.

2.8.1. Molecular Dynamic Simulations. The minimizations and MD simulations were performed using AMBER 12. Production for each of the four starting conformations was set to $100 \mathrm{~ns}$, for a cumulative sampling time of $400 \mathrm{~ns}$ for both labeled glycans. Energy minimizations through $500 \mathrm{k}$ cycles of steepest descent with a restraint of $5 \mathrm{kcal} / \mathrm{mol}^{*} \AA^{2}$ applied to all heavy atoms. For the 2-AB-labeled glycans, an additional $500 \mathrm{k}$ unrestrained minimization was carried out, to relax the acyclic GlcNAc conformation. Following the minimization stage(s), the system was heated from 5 to $298.5 \mathrm{~K}$ over 50 ps and then equilibrated at $300 \mathrm{~K}$ for 250 ps. This step was followed by a $100 \mathrm{~ns}$ production run. The resultant trajectory files were stripped of water using the ptraj module of AMBER 12. The trajectories were visualized using VMD v.1.9.3 beta $1,{ }^{21}$ and the highest populated conformers identified in terms of the torsion angle values and relative populations over $100 \mathrm{~ns}$.

2.8.2. Sequence Alignments and Homology Model. The $P D B e$ search engine available through the EMBL European Bioinformatics Institute (EBI) Web site was used to select protein structures with sequence identity higher than $30 \%$ relative to BKF (GenBank reference: AAI12589.1). Thermotoga maritima alpha fucosidase (TMF, 1ODU PDBid ${ }^{22}$ ) with $35.7 \%$ identity was selected as template, and two sequence alignments were used to build BKF homology models, one obtained with the program MODELER v.9.15, ${ }^{23}$ which was also used for the structure generation and scoring, and one with Clustal Omega. $^{24} \mathrm{~A}$ total of $10 \mathrm{BKF} 3 \mathrm{D}$ structures were visually analyzed by structural alignment to their TMF template using PyMol v.1.4.1. ${ }^{25}$ The most suitable structures were selected in terms of the conservation of the position of critical residues, such as the nucleophile and assisting base relative to the TMF template and the discrete optimized protein energy (DOPE) score.

2.8.3. MD Conformational Analysis of the AQCLabeled Fuc- $\boldsymbol{\alpha}(1-6)-$ GIcNAc. The conformations visited during the $100 \mathrm{~ns}$ production from one of the MDs can be described in terms of the values of the $\alpha(1-6)$ linkage $\psi, \varphi$, and $\omega$ torsions. A typical distribution is shown in SI Figure S-3 as an example. Conformational propensity was determined based on bins of width of $\pm 40^{\circ}$, centered around the average values of the highest populated conformers identified through graphs as the one shown in SI Figure S-3. The torsion angle values and relative populations of the four highest populated AQC-tagged sugars are shown SI Table S-3.

2.8.4. MD Conformational Analysis of the 2-ABLabeled Fuc- $\boldsymbol{\alpha}(1-6)-G I C N A C$. The same approach was taken to perform the conformational analysis of the 2-ABlabeled glycan. A typical distribution is shown in SI Figure S-4 as an example. The four highest populated conformers identified during the cumulative $400 \mathrm{~ns}$ simulation of the 2AB-labeled glycan are shown in SI Table S-4. All other conformers not included in the table have a population of less than $3 \%$.

2.8.5. Potential Structure of the 2-AB-Labeled Glycan in Complex with BKF. The structural alignment of the fucose cocrystallized with TMF to the ring atoms of the fucose of the highest populated conformers obtained through $\mathrm{MD}$ was used 


\section{A. 2-AB-labeled IgG $\mathbf{N}$-glycans}

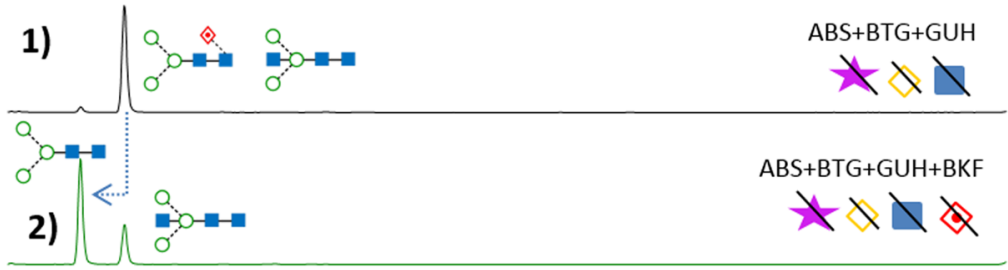

\section{B. RapiFluor-MS-labeled IgG $\mathbf{N}$-glycans}

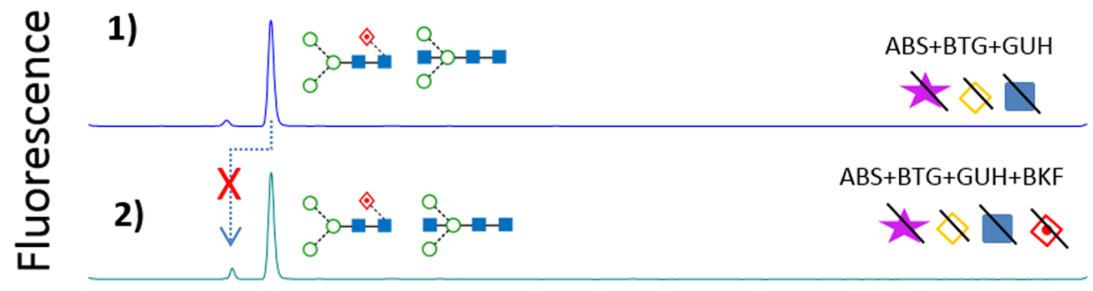

\section{AQC-labeled IgG $N$-glycans}

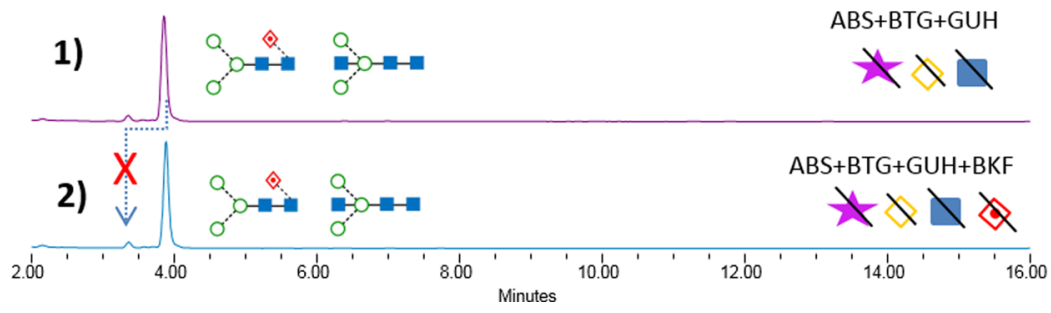

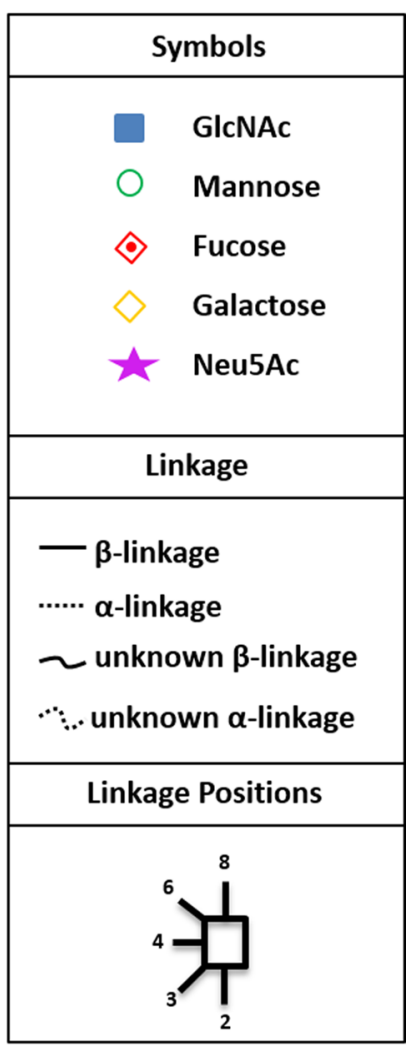

Figure 2. HILIC UPLC chromatograms showing comparison of core $\alpha(1,6)$ fucose removal by using BKF for (A) 2-AB, (B) RapiFluor-MS, and (C) AQC (aminoquinoline carbamate)-labeled IgG $N$-glycans previously digested with sialidase (ABS), galactosidase (BTG), and hexosaminidase (GUH). Efficient reduction of $\alpha(1-6)$ core linked fucose residues for 2-AB-labeled $N$-glycans is depicted with an arrow showing the reduction of glycan structure FM3 in panel A.1 to M3 shown in panel A.2. Inefficient reduction of $\alpha(1-6)$ core linked fucose residues for RapiFluor-MS- and AQC-labeled $\mathrm{N}$-glycans, with little/no reduction in the corresponding M3 glycan structure, are depicted with an arrow with a red cross for panels B and C. UPLC chromatograms showing the full exoglycosidase digestion arrays are found in SI Figure S-2. The experimental conditions for the exoglycosidase digestion were followed according to SI Table S-1. ABS $(\alpha 2-3,6,8,9$ Neuraminidase A, releases $\alpha(2-3,6,8,9)$ linked nonreducing terminal sialic acids), BTG $(\beta 1-3,4$ Galactosidase, releases $\alpha(1-3,4)$ linked nonreducing terminal galactose, GUH $(\beta$ - $N$-Acetylglucosaminidase S, releases $\beta(1-2,3,4,6)$ linked nonreducing $N$-acetyl glucosamine), and BKF $(\alpha 1-2,3,4,6$ Fucosidase, releases $\alpha(1-2,3,4)$ linked nonreducing terminal fucose. Colored Oxford nomenclature is used for glycan designation, and a legend is provided in the figure. ${ }^{12}$

as a method to establish the potential fit of the labeled glycan into the BKF binding site. As shown in SI Figure S-5 panels C and E, conformers 1 (40.3\% populated) and $3(6.7 \%$ populated) have the $2-\mathrm{AB}$ label pointing out of the active site, while conformers $2(36.5 \%)$ and $4(5.8 \%)$, shown in SI Figure S-5 panels D and F, have the label pointing toward the inside of the enzyme, suggesting that these conformation would not bind.

2.8.6. DFT Conformational Analysis of the AQCLabeled Fuc- $\boldsymbol{\alpha}(\mathbf{1 - 6 )}-\mathrm{GlcNAc}$. All DFT calculations were run with NWChem v.6.3, ${ }^{26}$ at the B3LYP/6-311G** level of theory. ${ }^{27}$ A DFT analysis of the relative stability of the different conformers identified through the MD simulation was carried out to verify the identity of such conformers as local energy minima and ultimately as a validation of the force field combination chosen to describe the fluorescently labeled glycans. During such analysis, we took into consideration that while the MD was ran in explicit solvent, all DFT calculations were ran in vacuo, which could alter considerably the relative stabilities of some conformations, due to the formation of internal hydrogen bonds, not populated (or relevant) in bulk water. $^{28}$

Calculations were run at the B3LYP/6-311G** level of theory. Conformer 2 from the MD simulations was not stable in vacuo, because the internal hydrogen bonds formed in the geometry optimization stabilizes the structure. The glycosidic bond has to rotate to allow this hydrogen bonding to occur. The DFT B3LYP/6-311G** global minimum corresponds to the highest populated conformer obtained through $\mathrm{MD}$, i.e. conformer $\mathbf{1}$. The difference in conformer 2 from $\mathrm{MD}$ and the optimized conformer (labeled conformer A) from DFT can be seen in SI Figure S-6. Conformers 1, 3, and 4 were identified as minima by DFT, and their relative energies are shown in SI Table S-5.

2.8.7. DFT Conformational Analysis of the 2-ABLabeled Fuc- $\alpha(1-6)-G I C N A c$. The DFT geometry optimization of the different conformers obtained for the 2-AB-labeled glycan through the MD simulations was carried out with the same protocol used in the case of the AQC-labeled glycan. Conformer 1 from MD simulations was not stable in vacuo, 
with the DFT B3LYP/6-311G** global minimum corresponding to a populated conformer identified from MD simulations, listed as conformer B in SI Table S-6. Conformer B has a relative population of 2.1. SI Figure S-7 shows the internal hydrogen bonding that stabilizes conformer $\mathbf{B}$ in vacuo and the difference in torsion angles between conformer $\mathbf{1}$ (from $\mathrm{MD}$ ) and conformer B. Conformers 2, 3, and 4 were identified as minima, and their relative energies were calculated, as shown in SI Table S-6.

\section{RESULTS AND DISCUSSION}

We first explored if the presence of aminoquinoline labels affected the performance of exoglycosidase arrays. RapiFluorMS- or AQC-labeled immunoglobulin G (IgG) N-glycans were sequenced using standard exoglycosidase digestion conditions and compared to 2-AB-labeled IgG $N$-glycans as a control (see experimental details and SI Figure S-1, Table S-1). As shown in Figure 2, negligible removal of core $\alpha(1-6)$ fucose was observed for RapiFluor-MS- and AQC-labeled IgG N-glycans, compared to 2-AB-labeled IgG N-glycans. In contrast, complete sialidase, galactosidase, and hexosaminidase reactions were observed for each of the three labels. Thus, only removal of core $\alpha(1-6)$ fucose by BKF was adversely affected by the presence of an aminoquinoline label.

Reaction conditions were optimized to create an exoglycosidase array fully capable of sequencing aminoquinoline-labeled $N$-glycans (including core $\alpha(1-6)$ fucose removal). This involved the use of sequential enzyme digests (SI Table S-2), higher BKF concentrations, and longer reaction times. Using this approach, it was possible to improve BKF removal of core $\alpha(1,6)$ fucose from AQC-labeled $N$-glycans (see experimental details and SI Figure S-2, Table S-2). However, this array procedure took 3-4 days to execute, compared to $\sim 18 \mathrm{~h}$ with 2-AB-labeled glycans. These data illustrate that inhibition of BKF by aminoquinoline labels is not exhaustive, suggesting that creation of BKF variants that more efficiently remove core $\alpha(1,6)$ fucose might be plausible. In the meantime, these conditions provide analysts a procedure for sequencing aminoquinoline-labeled $\mathrm{N}$-glycans in an extended protocol.

To understand the molecular basis for the reduced ability of BKF to remove core $\alpha(1,6)$ fucose from for AQC-labeled $N$ glycans, we used a combination of molecular simulation techniques, namely, molecular dynamics (MD), ab initio density-functional theory (DFT), and homology modeling, to study the recognition of the AQC- and 2-AB-labeled Fuc- $\alpha(1-$ $6)$-GlcNAc, designed to assess the structural complementarity of the BKF binding site with the labeled sugar conformations at $300 \mathrm{~K}$, as well as the intrinsic conformational flexibility of the labeled sugars. Because of the lack of structural data on $\mathrm{BKF}$, we built 3D models of the complexes with the AQC- and 2-ABlabeled minimum binding motif Fuc- $\alpha(1-6)-$ GlcNAc via homology modeling. The BKF model was based on the structure of T. maritima $\alpha$-L-fucosidase cocrystallized with fucose (PDBid 1ODU), ${ }^{23}$ with which BKF shares $36 \%$ sequence identity. ${ }^{24}$ The cocrystallized fucose was used as reference for the structural alignment of the AQC- and 2-ABtagged Fuc- $\alpha(1-6)$-GlcNAc conformers obtained through molecular dynamics (MD) in solution. We ran a collective 400 ns MD simulation for each labeled sugar, based on four independent and uncorrelated $100 \mathrm{~ns}$ trajectories at $300 \mathrm{~K}$ and 1 bar. Parameters for the were set based on a GLYCAM06 ${ }^{15}$ / $\mathrm{GAFF}^{16}$ force field scheme. The highest populated conformers were verified as energy minima by hybrid DFT (B3LYP) calculations. Further details on the computational method and data are included in the experimental section (see Computational Methods, SI Figures S-3-S-6, Tables S-3-S-6).

The conformational analysis of the unbound AQC-labeled Fuc- $\alpha(1-6)$-GlcNAc shows that only four conformers are significantly populated $(>10 \%)$ in solution, with the highest populated at $40 \%$, see SI Table S-3. These conformers are characterized by distinct values of the $\alpha(1-6)$ glycosidic linkage $\psi$ and $\omega$ angles, which determine the spatial orientation of the structurally rigid AQC group and of the O-4 on the GlcNAc residue where the $\mathrm{N}$-glycan GlcNAc-trimannose group are attached to, see Figure 3, panel A.
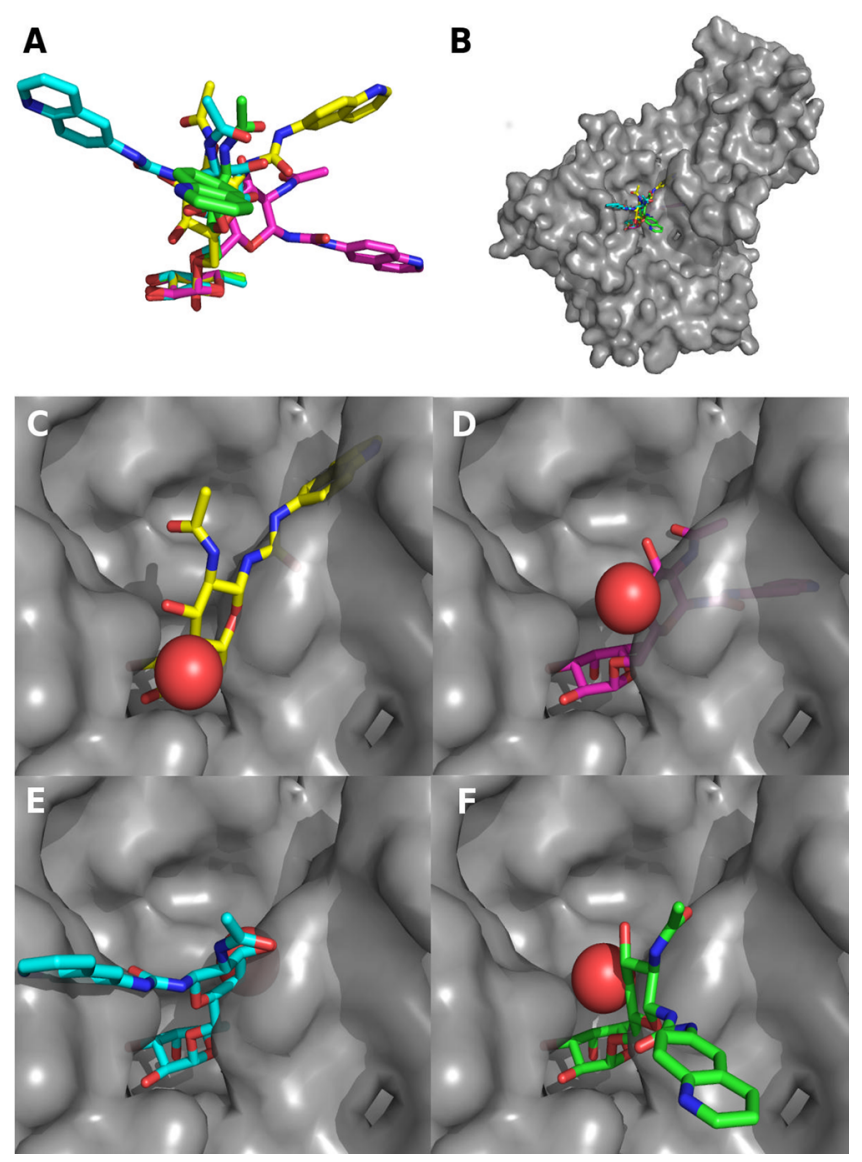

Figure 3. Panel A, structural alignment of the four highest populated conformers obtained from the analysis of a cumulative $400 \mathrm{~ns} \mathrm{MD}$ of the AQC-labeled Fuc- $\alpha(1-6)$-GlcNAc. Panel B, structural alignment of the four highest populated conformers on to the fucose in the BKF 3D model binding site. Panels C, D, E, and F, close up of the spatial orientation of the different conformers, with populations $40 \%$ (yellow), 25\% (purple), 11\% (cyan), and 10\% (green), respectively. The GlcNAc O4, where the $\mathrm{N}$-glycan chain is attached, is shown as a red sphere.

Structural alignment of the highest populated AQC-labeled conformers onto the fucose in the BKF binding site, shows that only the conformation with a relative population of $10 \%$ over $400 \mathrm{~ns}$, fits in the binding site without major steric clashes for the AQC tag and for the remainder of the sugar linked to the $\mathrm{O}-4$ on the GlcNAc residue. As shown in Figure $3 \mathrm{C}-\mathrm{F}$, because of the narrow structure of the BKF binding site, the three highest populated conformers are a very tight fit, not only for the AQC tag but also for the remainder of the glycan at position $\mathrm{O} 4$ of GlcNAc residue. The relative populations 
obtained through $\mathrm{MD}$ suggest that between the optimally fitting (10\%) and the highest populated conformer (40\%), the free energy $(\Delta G)$ barrier is relatively low, i.e. $\Delta G=+3.4 \mathrm{~kJ} / \mathrm{mol}$ $(1.4 \mathrm{kT})$. This is in agreement with the experimental observation that digestion can be brought to completion by stressing the reaction conditions, for example, through prolonged reaction time or increased fucosidase concentrations. In case of the 2-AB-labeled sugar, because of the characteristic acyclic structure of the GlcNAc, the conformational dynamics visits only two significantly populated conformations through 400 ns, see SI Table S-2, with comparable relative populations, i.e. $40 \%$ and $36 \%$. Only one of the two has a near perfect fit in the BKF binding site, and the almost equal population distribution, due to the intrinsic flexibility of the acyclic GlcNAc, corresponds to $\Delta G=+0.3 \mathrm{~kJ} / \mathrm{mol}(0.5 \mathrm{kT})$, suggesting a high degree of conformational flexibility at room temperature that would facilitate substrate recognition and binding.

\section{CONCLUSIONS}

Biochemical evidence is provided that core $\alpha(1,6)$ fucose removal in the presence of aminoquinoline labels such as AQC or RapiFluor-MS for glycan characterization is hindered using standard exoglycosidase digestion conditions. Optimized experimental conditions using high concentrations of BKF and longer reaction times are presented to show core $\alpha(1,6)$ fucose removal in the presence of AQC. This biochemical data is supported by our computational model that suggest a rationale for the poor ability of BKF to remove $\alpha(1,6)$ fucose from aminoquinoline labeled $\mathrm{N}$-glycans. This rationale is essentially based on the low degree on intrinsic flexibility of the aminoquinoline group. MD simulations show that only four distinct conformations of the AQC-labeled sugar are significantly populated in solution $(>10 \%)$. Based on the structure of $\mathrm{BKF}$ obtained from homology modeling, only one of these conformers fits in the binding site without major steric clashes with the protein. We acknowledge that such experimental conditions may not be suitable in analytical applications where cost and/or time are significant factors. Thus, future work will involve attempts to improve BKF's ability to hydrolyze core fucose from aminoquinoline-labeled $\mathrm{N}$-glycans using structureguided mutagenesis strategies.

\section{ASSOCIATED CONTENT}

\section{Supporting Information}

The Supporting Information is available free of charge on the ACS Publications website at DOI: 10.1021/acs.jproteome.7b00580.

HILIC UPLC chromatograms for standard and optimized exoglycosidase digestion arrays; distribution of $\varphi$ (blue), $\psi$ (red), and $\omega$ (green) values of the AQC- and 2AB-labeled glycan from each of the four $100 \mathrm{~ns} \mathrm{MD}$ simulations; structural alignment of highest populated conformers of 2-AB-labeled Fuc- $\alpha(1,6)$-GlcNAc cocrystallized with TMF; conformer 2 (yellow) and conformer A (blue) of AQC-labeled Fuc- $\alpha(1-6)$-GlcNAc, aligned at the fucose residue; conformer 1 (pink) and conformer $\mathrm{B}$ (yellow) of 2-AB-labeled Fuc- $\alpha(1-6)$-GlcNAc, aligned at the fucose residue; exoglycosidase digestion array panels for standard and optimized conditions; conformational analyses for AQC- and 2-AB-labeled Fuc- $\alpha(1-6)$ -
GlcNAc; torsion angles values and relative energies for AQC- and 2-AB-labeled Fuc- $\alpha(1-6)$-GlcNAc (PDF)

\section{AUTHOR INFORMATION}

\section{Corresponding Author}

*Tel: 00353-1215 8154. E-mail: roisin.oflaherty@nibrt.ie. ORCID 10

Róisín O’Flaherty: 0000-0003-1941-4775

Elisa Fadda: 0000-0002-2898-7770

\section{Author Contributions}

R.O.F and P.M.R. developed the idea, designed the experiments for the study and wrote the manuscript with the help of A.M.H, C.H.T. and E.F. The preparation of IgG $N$-glycans labeled with $2-\mathrm{AB}$ and $\mathrm{AQC}$, exoglycosidase digestions and UPLC chromatography was performed by R.O.F and the molecular modeling was performed by A.M.H. and P.J.H, with this portion of the research directed by E.F. All authors participated in discussions and proof-reading the manuscript.

Notes

The authors declare the following competing financial interest(s): C.T. is a researcher at New England Biolabs, a commercial supplier of glycosidases.

\section{ACKNOWLEDGMENTS}

R.O.F. and P.M.R. gratefully acknowledge the financial support from EU FP7 program HighGlycan, grant no. 278535, and SFI Spokes program Microbe Mom, grant no. 16/SP/3827 for funding this work. A.H. gratefully acknowledges Maynooth University for funding through the John and Pat Hume Scholarship Scheme. E.F. gratefully acknowledges the Irish Centre for High-End Computing (ICHEC) for the generous allocation of computational resources.

\section{ABBREVIATIONS}

PNGase F, Peptide- $N$-Glycosidase F; 2-AB, 2-aminobenzamide; UPLC, ultra performance liquid chromatography; Gal, galactose; GlcNAc, glucosamine; AQC, 6-aminoquinolyl- $N$ hydroxysuccinimidyl carbamate; IgG, immunoglobulin G; ABS, $\alpha(2-3,6,8,9)$ Neuraminidase A; BTG, bovine testes $\beta(1-3 / 4)$ galactosidase; GUH, $\beta$-N-Acetylglucosaminidase S; BKF, $\alpha(1-$ $2,3,4,6)$ Fucosidase; JBM, $\alpha(1-2,3,6)$-Mannosidase; $\mathrm{NaCNBH}_{3}$, sodium cyanoborohydride; $\mathrm{AcOH}$, acetic acid; $\mathrm{DMSO}$, dimethyl sulfoxide; $\mathrm{MeCN}$, acetonitrile; $\mathrm{MD}$, molecular dynamics; PDB, protein data bank; TMF, Thermotoga maritima alpha fucosidase; DFT, density functional theory

\section{REFERENCES}

(1) Staudacher, E.; Altmann, F.; Wilson, I. B.; et al. Fucose in Nglycans: from plant to man. Biochim. Biophys. Acta, Gen. Subj. 1999, 1473 (1), 216-236.

(2) Becker, D. J.; Lowe, J. B. Fucose: biosynthesis and biological function in mammals. Glycobiology 2003, 13 (7), 41R-53R.

(3) Mimura, Y.; Katoh, T.; Saldova, R.; et al. Glycosylation engineering of therapeutic IgG antibodies: challenges for the safety, functionality and efficacy. Protein Cell 2017, DOI: 10.1007/s13238017-0433-3.

(4) Stockmann, H.; O’Flaherty, R.; Adamczyk, B.; et al. Automated, high-throughput serum glycoprofiling platform. Integr Biol. (Camb) 2015, 7 (9), 1026-32.

(5) Zhang, L.; Luo, S.; Zhang, B. Glycan analysis of therapeutic glycoproteins. MAbs 2016, 8 (2), 205-15. 
(6) Marino, K.; Bones, J.; Kattla, J. J.; et al. A systematic approach to protein glycosylation analysis: a path through the maze. Nat. Chem. Biol. 2010, 6 (10), 713-23.

(7) Walsh, I.; O’Flaherty, R.; Rudd, P. M. Bioinformatics applications to aid high-throughput glycan profiling. Perspectives in Science 2017, 11, 31-9.

(8) Arnold, J. N.; Wormald, M. R.; Suter, D. M.; et al. Human serum IgM glycosylation: identification of glycoforms that can bind to mannan-binding lectin. J. Biol. Chem. 2005, 280 (32), 29080-7.

(9) Houel, S.; Hilliard, M.; Yu, Y. Q.; et al. N- and O-glycosylation analysis of etanercept using liquid chromatography and quadrupole time-of-flight mass spectrometry equipped with electron-transfer dissociation functionality. Anal. Chem. 2014, 86 (1), 576-84.

(10) Stockmann, H.; Duke, R. M.; Millan Martin, S.; et al. Ultrahigh throughput, ultrafiltration-based n-glycomics platform for ultraperformance liquid chromatography (ULTRA(3)). Anal. Chem. 2015, 87 (16), 8316-22.

(11) Qing, Y. Y. Applying a Novel Glycan Tagging Reagent, RapiFluor-MS, and an Integrated UPLC-FLR/QTof MS System for Low Abundant N-Glycan Analysis. Application Note 2015; 720005383EN:Waters. Avilable at the following: http://www.waters. com/webassets/cms/library/docs/720005383en.pdf

(12) Harvey, D. J.; Merry, A. H.; Royle, L.; et al. Proposal for a standard system for drawing structural diagrams of $\mathrm{N}$ - and O-linked carbohydrates and related compounds. Proteomics 2009, 9 (15), 3796801.

(13) Stöckmann, H.; Duke, R. M.; Millán Martín, S.; et al. Ultrahigh Throughput, Ultrafiltration-Based N-Glycomics Platform for Ultraperformance Liquid Chromatography (ULTRA 3). Anal. Chem. 2015, 87 (16), 8316-22.

(14) Royle, L.; Campbell, M. P.; Radcliffe, C. M.; et al. HPLC-based analysis of serum $\mathrm{N}$-glycans on a 96 -well plate platform with dedicated database software. Anal. Biochem. 2008, 376, 1-12.

(15) Kirschner, K. N.; Yongye, A. B.; Tschampel, S. M.; et al. GLYCAM06: a generalizable biomolecular force field. Carbohydrates. J. Comput. Chem. 2008, 29 (4), 622-55.

(16) Wang, J.; Wolf, R. M.; Caldwell, J. W.; et al. Development and testing of a general amber force field. J. Comput. Chem. 2004, 25 (9), $1157-74$.

(17) Schrödinger, LLC. Schrödinger Maestro v.10.7.015; Schrödinger: New York, 2012.

(18) Pettersen, E. F.; Goddard, T. D.; Huang, C. C.; et al. UCSF Chimera-a visualization system for exploratory research and analysis. $J$. Comput. Chem. 2004, 25 (13), 1605-12.

(19) Case, D. A., Darden, T. A., Cheatham, T. E. et al. AMBER 12; University of California: San Fransisco, 2012.

(20) Jorgensen, W. L.; Chandrasekhar, J.; Madura, J. D.; et al. Comparison of Simple Potential Functions for Simulating Liquid Water. J. Chem. Phys. 1983, 79 (2), 926-35.

(21) Humphrey, W.; Dalke, A.; Schulten, K. VMD: visual molecular dynamics. J. Mol. Graphics 1996, 14 (1), 33-8.

(22) Sali, A.; Blundell, T. L. Comparative protein modelling by satisfaction of spatial restraints. J. Mol. Biol. 1993, 234 (3), 779-815.

(23) Sulzenbacher, G.; Bignon, C.; Nishimura, T.; et al. Crystal structure of Thermotoga maritima alpha-L-fucosidase. Insights into the catalytic mechanism and the molecular basis for fucosidosis. J. Biol. Chem. 2004, 279 (13), 13119-28.

(24) Sievers, F.; Wilm, A.; Dineen, D.; et al. Fast, scalable generation of high-quality protein multiple sequence alignments using Clustal Omega. Mol. Syst. Biol. 2011, 7, 539.

(25) Schrödinger, LLC. The PyMOL Molecular Graphics System, Version 1.4.1; Schrödinger: New York, 2012.

(26) Valiev, M.; Bylaska, E. J.; Govind, N.; et al. NWChem: A comprehensive and scalable open-source solution for large scale molecular simulations. Comput. Phys. Commun. 2010, 181 (9), 147789.

(27) Stephens, P. J.; Devlin, F. J.; Chabalowski, C. F.; et al. Ab-Initio Calculation of Vibrational Absorption and Circular-Dichroism Spectra
Using Density-Functional Force-Fields. J. Phys. Chem. 1994, 98 (45), 11623-7.

(28) Kirschner, K. N.; Woods, R. J. Solvent interactions determine carbohydrate conformation. Proc. Natl. Acad. Sci. U. S. A. 2001, 98 (19), 10541-5. 